\title{
A Survey on Image Inpainting Techniques to Reconstitute Remotely Sensed Images
}

\author{
Rohini B. Late ${ }^{1}$, N. G. Dharashive ${ }^{2}$ \\ ${ }^{1}$ SRTM University, Department of CSE, M. S. Bidve Engineering College, Latur, Maharashtra, India
}

\begin{abstract}
Modification of digital image is general practice with various intentions now days because of extensive availability of sophisticated image editing tools. Image inpainting is one of the approaches to recuperate lost or damaged part of image in order to make it visually plausible and restore its unity. Nonresponsive pixel (dead pixel) or removing unwanted objects from digital image is frequently preferred in the application of remote sensing. Remotely sensed images are suffered due to stripping and dead pixels. To solve this problem many image inpainting techniques are proposed to recover damaged or stripping images. In this paper we provide a survey of different techniques used for remotely sensed image inpainting like Exampler based, PDE based, Structure based, Texture based etc.
\end{abstract}

Keywords: Image Inpainting, Image Reconstruction (gap filling), Satellite image, Landsat7 SLC-off reconstruction.

\section{Introduction}

Inpainting is the art of restoring lost part of an image and reconstructing it based on background image information. Digital image inpainting tries to imitate this process and perform inpainting automatically. In image inpainting we would like to create original image but it is absolutely not viable without the prior knowledge of image. In case of digital images, only the available image is taken for the experiment and thus filling in a hole(dead pixel) that cover a whole object. It is not possible to replace that whole object based on the required current information[1].Therefore the aim of image inpainting is not to recover the original image but to create visually plausible image in such a way that it is not detectable by casual(ordinary) observer who is not familiar with original image. Application of image inpainting ranges from restoration (rebuilding) of old films, photograph and paintings, to object removal in digital photographs such as text, subtitles stamp. It is also applied to red-eye correction, super resolution, compression, image coding and transmission etc. Inpainting in satellite images required for filling gaps occurs due to instrumentation error, losses of image data during transmission; such area involve reconstruction in satellite image referred as gapped area (dead pixels)in remotely sensed (satellite) images.

The Landsat7 Scan Line Corrector (SLC), is an electromechanical device designed to compensate for the forward motion of the satellite during(Enhanced Thematic Mapper Plus) ETM + scanning, has failed permanently on May 31, 2003 , with the SLC now turned off, the ETM+ is losing $22 \%$ of data due to increased scan gap[2][3], hence, the images which acquired before the failure of SLC are designated SLC-on images and those acquired after the failure of SLC are designated SLC-off images[4].In spite of the SLC failure produces, obviously negative impact on Landsat7 ETM+ imagery fortunately, the SLC failure had no effect on the radiometric and geometric quality of ETM+ sensor and about $80 \%$ of pixels in the image scanned perfectly. So the ETM+ still significant for some applications and for users over other costly alternative [5],[6].
Many algorithms have been proposed for gap filling in Landsat7 SLC-off images. Proposed algorithm for Landsat7 SLC-off image inpainting can be characterized as single source, multi source and hybrid. The first category mostly is based on within image pixel similarities/dissimilarity rules. In the second category gap areas are constructed using other useful sources. Hybrid techniques make combination of both single source and multisource image inpainting techniques.

\section{Image Inpainting For Remotely Sensed Images}

In spite of collapse Landsat7 imagery, the SLC-off images retain its significant utility for scientific research and application. Hence many methodologies have been proposed to determine SLC-off problem by finding suitable algorithms to estimate the reflectance values at un scanned pixels precisely and robustly [7].

\subsection{Single Source Method}

In single source category the gapped (dead pixels) areas are constructed using non-gapped areas(remaining part of image) in the image itself. Following computational techniques can be used.

\section{(a) Averaging}

In averaging method line drop is conquer by replacing zero value by means of all the pixels in previous and following lines. For modern detector stripping is along with column instead of row. E.g. If line 2 is corrected by averaging of line 1 st and line 3 rd value. [8].

\section{(b) Simple Interpolation}

In simplest case replacement the missing pixel value by value calculated from neighboring pixels values. Nearest neighbor, bilinear, bicubic and other interpolation methods are used for gap filing in ETM+ SLC-off images using weighted average . In general the performance of such interpolation algorithms was inaccurate due to their simple and easy implementation because the interpolated value was calculated from pixels 


\section{International Journal of Science and Research (IJSR) \\ ISSN (Online): 2319-7064}

Index Copernicus Value (2013): 6.14 | Impact Factor (2014): 5.611

surrounding the gap locations rather than estimated the reflectance of land [9].

\section{(c) Spectral Interpolation.}

To fill the image gaps using coincident spectral data from the SLC-off image itself, the multi scale segmentation model derived from complete satellite images (SLC-on) was overlaid on SLC-off image, then the missing data were estimated using the spectral data on SLC-off that intersect with segment boundary model[10]. Maxwell presented a development of multi-scale segmentation approach for interpolating the ETM+SLC-off images depending on three assumptions related to segmentation based algorithm:

1)The spectral value of adjacent pixels is probably same more than non- adjacent pixels.

2)Pixels have similar spectral values within a specific landscape unit, like crop, grass fields or group of trees.

3)In general for several years no changeable in small landscape unites.

The quality of the segment model depends on some conditions that available in the image which is used to generate the segment model:

1) The segment image has minimum cloud cover.

2) The image selected with appropriate time of the year (i.e. inappropriate time will not provide precise separation of landscape units).

3) The time interval between segment image and SLC-off image (long time interval, more change in landscape structure).

Three scale segment model (scales 20, 15 and 10), derived from SLC-on image, were used to direct the spectral interpolation over the gaps in SLC-off image. A comparison between original Landsat5 and filled images was used to evaluate the accuracy of the gap filled technique, the results indicate that most of gap pixels with high accuracy were interpolated using segment model of scale 10, thus the product could be used in land cover mapping (e.g. forest and grass), crop mapping and regional-scale studies. At the same time, at pixel level the accuracy of the reflectance prediction was low especially in narrow and tiny objects as in roads and rivers[4],[11], [12].

\subsection{Multi Source Method}

In the Multi source gap filling methods areas are constructed using other image of same scene that is gapped images can be filled with other image of same satellite or other image of other satellite[2][13][14].

Images of Landsat7 after failure of SLC taken are known as SLC-off images which contains dead pixel. LLHM( localized linear histogram match): The pixel value of SLC-off images to be filled by calculating corrective gain and bias adjustment using moving window around each pixel in SLCoff scene to obtain localized linear transformation function and then transform pixel value of SLC-on image to fill in damaged area in SLC-off image this is basis of LLHM, known as phase one method for gap filling in SLC-off image in LANDSAT 7 image[15].
AWLHM( Adaptive Window Local Histogram Matching): In AWLHM phase 2 is enhancement to phase 1 techniques for gap filling in LANDSAT7 SLC-off images that allow user to choose one or more SLC-off image because location of gaps differ per image which taken for same area[16][17].

Adaptive Window Local Histogram Matching (AWLHM) algorithm was based on the same concept as LLHM with exclusion in the change of the size of moving window. For each moving window the gain and bias were found statistically from effective pixels that was used for estimating each un-scanned pixels. The size of moving widow changed depending on the distribution of commonly scanned pixel. Other satellite images can be used to reconstruct image which was proposed in [14]. In this paper author uses CBERS-02B data for gap filling in Landsat7 SLC-off images. They have used same algorithms proposed in phase 1, phase 2 gap filling algorithm by USGS(United States Geological Survey), but variation is instead of using same satellite data as reference image they have used another satellite image as reference scene and they have used replacement method In this replacement method gap value in SLC-off images of Lansat7 is replaced by corresponding value of CBERS-02B values. Reference [14] shows that AWLHM give more accurate result if computational speed is ignored. If computational speed is more important than statistical error LLHM should be preferred.

Q. Huang and others[9] used multi temporal images to reconstruct SLC-off ETM+ images by applying the Local Correlation Analysis approach (LCA) on simulated and real SLC-off ETM+ images and match up to results with applying the LLHM method on same tested images. Visual and statistical indices indicated that the LCA approach filled image coincided(matched) well with the target image and also preserving the spatial continuity in vegetation and soil land cover[9].

In (Principal Component Transformation) PCT-gap fill methodology the basic idea is to fill gap areas in spectral images by the help of valuable pixels from the earlier obtained ETM+ as auxiliary images and compare results with LLHM . For an illustration let FI and VA be the Fill Image and the valuable Area for fill gaps, GA and NGA be the Gapped Area and Non-Gapped Area from the Gap Image GI.

The procedure of which will follow the next steps

a) Pixels in VA numerically adaptation to pixels in NGA.

b)Using NGAs for calculation the needed statistics for Inverted PCT (IPCT), i.e. the empirical mean, correlation matrix and Eigen values.

c) VAs conversion to Transformed VAs (TVAs) using the principal component transformation.

d)Inversely transformation of the TVAs using the obtained statistics from step (2) to make the New VAs (NVAs).

e) Replacing the NVAs to the GA to form a econstructed Image (RI). [2]

\subsection{Hybrid Method}

Hybrid method is combination of single source and multisource method for filling gaps in satellite images. 


\section{International Journal of Science and Research (IJSR)}

ISSN (Online): 2319-7064

Index Copernicus Value (2013): 6.14 | Impact Factor (2014): 5.611

\section{Inpainting Algorithms}

In the field of digital image processing number of algorithms have been proposed for image reconstruction known as inpainting algorithms e.g. Bertalmio's inpainting algorithm, Oliveira's inpainting algorithm, Criminisi's algorithm etc. Here idea is to apply inpainting algorithm for inpainting of remotely sensed images and it is categorized it into following categories: PDE based Inpainting Exemplar based Inpainting, Texture based Inpainting, Structure based inpainting and Hybrid based Inpainting.

\subsection{PDE based Inpainting}

Partial Differential Equation (PDE) based algorithm is proposed by Bertalmio et.al [1]. This algorithm is the iterative algorithm. The algorithm is to continue geometric and photometric information that arrives at the border of the occluded area into area itself. This is done by propagating the information in the direction of minimal change using "isophote lines". This algorithm will produce good results if missed regions are small one. But when the missed regions are large this algorithm will take so long time and it will not produce good results Then inspired by this work, Chan and Shen [18] proposed the Total Variational (TV) Inpainting model. This model uses Euler-Lagrange equation and anisotropic diffusion based on the strength of the isophotes. This model performs reasonably well for small regions and noise removal applications. But the drawback of this method is that this method neither connects broken edges nor fill texture patterns. The TV model then extended to CDD (Curvature Driven Diffusion) [19] model. In which it included the curvature information of the isophotes to handle the curved structures in a better manner. Then telea in [20] propose a fast marching method. This is considered as a PDE method which is faster and simpler to implement than other PDE based algorithms. All of the above mentioned algorithms are very time consuming and have some problems with the damaged regions with a large size. PDE based technique has been widely used in number of applications such as image segmentation, restoration etc. These algorithms were focused on maintaining the structure of the Inpainting area,and hence these algorithms results blurred resulting image. Another drawback of these algorithms is that the large textured regions are not well reproduced.

\subsection{Exemplar based Inpainting.}

This method of image Inpainting is an important approach for reconstructing large target regions and proved to be very effective

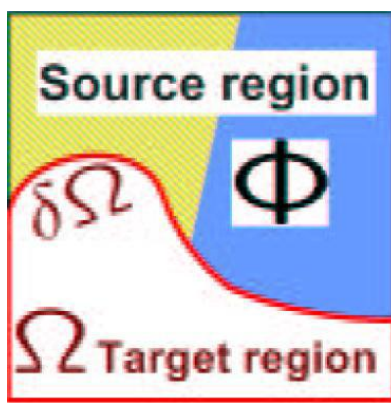

a

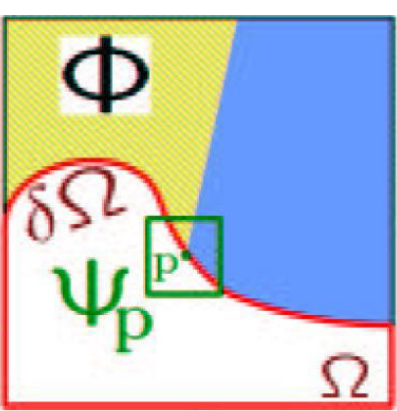

b
Figure 1: Structure propagation by exemplar-based texture synthesis.(a) Original image, with the target region $\Omega$, its contour $\delta \Omega$ and the source region $\Phi$ clearly marked. (b) We want to synthesize the area delimited by the patch $\psi \mathrm{p}$ centred on the point $\mathrm{p} € \delta \Omega$.( A. Criminisi, et al,2003)[21]

Basically it consists of two basic steps: in the first step priority assignment is done and the second step consists of the selection of the best matching patch. The exemplar based approach samples the best matching patches from the known region, whose similarity is measured by certain metrics, and pastes into the target patches in the missing region.

Exemplar-based Inpainting approach iteratively synthesizes the target (unknown)region by most similar patch in the source region.

Generally, an exemplar-based Inpainting algorithm includes the following four main steps:

1)Initializing the Target Region, in which the initial missing areas are extracted and represented with appropriate data structures.

2)Computing Filling Priorities, in this a predefined priority function is used to compute the filling order for all unfilled pixels $p € \delta \Omega$ in the beginning of each filling iteration.

3) Searching Example and Compositing, in which the most similar example is searched from the source region $\Phi$ to compose the given patch, $\Psi$ (of size $\mathrm{N} \times \mathrm{N}$ pixels) that centered on the given pixel $p$.

4)Updating Image Information, in which the boundary $\delta \Omega$ of the target region $\Omega$ and the required information for computing filling priorities are updated.

Number of algorithms are developed for the exemplar based image Inpainting. Such as, Criminisi developed an efficient and simple approach to encourage filling from the boundary of the missing region where the strength of isophote nearby was strong, and then used the sum of squared difference (SSD) to select a best matching patch among the candidate source patches. In this algorithm of criminisi the region filling order is determined by the priority based mechanism.[21]

Most of the Exemplar-based algorithms adopt the greedy strategy, so these algorithms suffer from the common problems of the greedy algorithm, being the filling order (priority)is very critical.

Exemplar based Inpainting will produce good results only if the missing region consists of simple structure and texture. 


\section{International Journal of Science and Research (IJSR) \\ ISSN (Online): 2319-7064}

Index Copernicus Value (2013): 6.14 | Impact Factor (2014): 5.611

And if there are not sufficient samples in image then it is impractical to synthesize the desired image.

\subsection{Texture Synthesis Based Inpainting}

The Texture synthesis based algorithms are used to complete the missing regions using similar neighbour hoods of the damaged pixels. It is one of the earliest methods of image Inpainting texture synthesis algorithms synthesizing the new image pixels from an initial seed, and then strives to preserve the local structure of the image. All the earlier Inpainting techniques utilized these methods to fill the target region by sampling and copying pixels from the neighbouring area. For e. g, Markov Random Field (MRF) is used to model the local distribution of the pixel. And new texture is synthesized by querying existing texture and finding all similar neighbourhoods. Their differences exist mainly in how continuity is maintained between existing pixels and Inpainting hole.[21]

The main objective of texture synthesis based inpainting is to generate texture patterns, which is similar to a given sample pattern. These methods can fill large textured regions, but depends on user choices on sampling location and content.

Texture synthesis approaches can be categorized into three categories:

a)Statistical (parametric)

b)Pixel-based (non-parametric).

c) Patch-based (non-parametric).

Statistical methods are more likely to succeed in reproducing irregular textures, but usually fail to reproduce structured/regular textures. On the other hand, pixel-based methods "build" on the sample texture pixel-by-pixel instead of applying filters on it, and their final outputs are of better quality than those of statistical methods, but they usually fail to grow large structured textures. Finally, patch-based [22] methods "build" on a sample texture patch-by-patch as opposed to pixel-by-pixel, thus they yield faster and more plausible regular textures.

The texture synthesis based Inpainting perform well in approximating textures. These algorithms have difficulty in handling natural images as they are composed of structures in form of edges. Also they have complex interaction between structure and texture boundaries. In some cases, they also require the user to specify what texture to replace and where to be replaced. Hence while appreciating the use of texture synthesis techniques in Inpainting, it is important to understand that these methods address only a small subset of Inpainting issues and these methods are not suitable for a wide variety of applications.

\subsection{Structure-based Inpainting}

Structure-based inpainting: in [23] present a sketch guided texture based method. Their approach first generates the sketch (i.e. first it reconstructs edges that separate smooth or texture regions), then guided by the sketch lines the information surrounding sketch lines is restored by patchbased matching algorithm. Finally under constrained of information surrounding sketch lines all of missed regions is reconstructed by exemplar based method. Rares and others [24] present a model very similar to that they use of level lines based algorithm instead of sketch based and apply higher level features extracted from the image.

\subsection{Hybrid based inpainting}

Hybrid inpainting technique is also called as Image Completion. It is used for filling large hole and preserves both structure and texture in a visually plausible manner. The hybrid approaches combine both texture synthesis and PDE based Inpainting for completing the holes. The main idea behind these approaches is that it decomposed the image into two separate parts, Structure region and texture regions. The corresponding decomposed regions are filled by edge propagating algorithms and texture synthesis techniques. These algorithms are computationally intensive unless the fill region is small. One important direction we believe is more natural to the inpainting process is by structure completion through segmentation. This technique uses a two-step approach: structure completion followed by texture synthesis. In the structure completion stage, segmentation is performed based on the color and texture information on the input and then the partitioning boundaries are extrapolated to generate a complete segmentation for the input .The second step consists of synthesizing texture and color information in each segment.

\section{Conclusion}

In this paper we review approaches on the existing techniques of image Inpainting. We discussed a variety of image Inpainting techniques such as, PDE based Inpainting, Exemplar based Inpainting, Texture based Inpainting, Structure based inpainting and Hybrid based Inpainting. A number of shortcomings and limitations were highlighted of these techniques.

It is observed that the PDE based Inpainting algorithms cannot fill the large missing region and it cannot restore the texture pattern. The theoretical analysis proved that exemplar based Inpainting will produce good results for Inpainting the large missing region also these algorithms can inpaint both structure and textured image as well. But they work well only if missing region consists of simple structure and texture.

Various gap filling method for satellite image and inpainting algorithm exists, Multi-source gap filling suffers from sharp radical changes in to images such as sun glint change, snow, cloud etc. In single-source inpainting algorithm if it can reconstruct large area it takes more computational time. There is need of powerful method which can reconstruct large as well as small area in less computational time to fill gaps.

\section{References}

[1] Marcelo Bertalmio, Luminita Vese, Guillermo Sapiro(2003), Simultaneous Structure and Texture 


\section{International Journal of Science and Research (IJSR) \\ ISSN (Online): 2319-7064}

Index Copernicus Value (2013): 6.14 | Impact Factor (2014): 5.611

Image Inpainting, IEEE transactions on image processing, vol. 12 , no. 8 .

[2] A. D. Boloorani, S. Erasmi, and M. Kappas, "MultiSource Remotely Sensed Data Combination: Projection Transformation Gap-Fill Procedure," Sensors, vol. 8, no. 7, Jul. 2008, pp. 4429-4440.

[3] M. J. Pringle, M. Schmidt, and J. S. Muir, "Geostatistical interpolation of SLC-off Landsat ETM+ images," ISPRS J. Photogramm. Remote Sens., vol. 64, no. 6, Nov. 2009,pp. 654-664.

[4] J. Chen, X. Zhu, J. E. Vogelmann, F. Gao, And S. Jin, "A simple and effective method for Filling gaps in Landsat ETM+ SLC-off Images," Remote Sens. Environ.,Elsevier Inc., Vol. 115, no. 4, Apr. 2011, pp. 1053-1064.

[5] F. Chen, x. Zhao, and h. Ye, "making use Of the landsat 7 slc-off etm + image Through different recovering Approaches" . In z. Karakehayov.: data Acquisition applications: cha.13,2012.

[6] F. Chen, L. Tang, C. Wang, and Q. Qiu, "Recovering of the thermal band of Landsat 7 SLC-off ETM+ image using CBERS as Auxiliary data,", Adv. Sp. Res., Elsevier, vol. 48, no. 6, Sep. 2011, pp. 1086-1093.

[7] T. K. Alexandridis, I. Cherif, C. Kalogeropoulos, S. Monachou, K. Eskridge, And N. Silleos, "Rapid error assessment for Quantitative estimations from Landsat 7 gapfilled Images," Remote Sens. Lett., vol. 4, no. 9, Sep. 2013, pp. 920-928.

[8] P. Nag and M. Kudrat, Digital Remote Sensing,1st ed., New Delhi:Concept Publishing Company, 1998, ch.6, pp. 111

[9] W. Hu, M. Li, Y. Liu, Q. Huang, and K. Mao, “A New Method of Restoring ETM + slcoff Images Based on Multi-temporal Images,", IEEE, no. 41001046, 2011, pp. 1-4.

[10] S. Maxwell, "Filling Landsat ETM+ SLC-off Gaps Using a Segmentation Model Approach," ,Photogrammetric Engineering \& Remote Eensing, October 2004, pp. 1109-111.

[11]C. Zeng, H. Shen, and L. Zhang, "Recovering Missing pixels for Landsat ETM+ SLC-off Imagery using multitemporal regression Analysis and a regularization method," Remote Sensing of Environment, vol. 131. Elsevier Inc., Apr-2013, pp. 182-194.

[12] S. K. Maxwell, G. L. Schmidt, and J. C. Storey, "A multi-scale segmentation approach To filling gaps in Landsat ETM+SLC-off Images," Int. J. Remote Sens., vol.28, no.23,Dec. 2007, pp. 5339-5356.

[13] USGS.(July-2004). SLC-off Gap-Filled Products Gapfill Algorithm methodology: Phase2 Gap-fill Algorithm [Online]. Available:http://landsat.usgs.gov

[14]F. Chen, L. Tang, and Q. Qiu, "Exploitation of CBERS02B as Auxiliary data in recovering the Landsat7 ETM+ SLC-off image," in Proc. 18th International Conf. Geoinformatics, Beijing, June 2010, pp. 1-6

[15]P. Scramuzza, E. Micijevic, and G. Chander, (2004). "SLC gap-filled Products: Phase one methodology [Online].

Available:http://www.landsat.usgs.gov/data_products/slc off.

[16] $\overline{\mathrm{S}}$. Gilbert, "Project Report : Gap-Filling Landsat 7 Imagery A study of techniques for Filling the gaps in
Landsat 7 imagery post- 2003 Purpose : Area of Study :" 2012

[17]USGS \& NASA." SLC-off Gap-Filled Products Gap-fill Algorithm Methodology: Phase 2", October 2004. Gapfill Algorithm Available from Www.ga.gov.au/servlet/bigobjfilemanager? Bigobjid=GA4861 (accessed on 2013).

[18] T. Chan and J. Shen, "Local inpainting models and TV inpainting," SIAM Journal on Applied Mathematics, Vol. 62, 2001, pp. 1019-1043.

[19]T. Chan and J. Shen, "Nontexture inpainting by curvature-driven diffusions," Journal of Visual Communication and Image Representation, Vol. 4, 2001, pp. 436-449

[20] Telea,"An Image Inpainting Technique Based On The Fast Marching Method", Journal Of Graphics Tools, Vol.9, No. 1, ACMPress 2004.

[21] A. Criminisi, P. Perez, and K. Toyama, "Region filling and object removal by exemplar- based image inpainting," IEEE Transactions on Image Processing, Vol. 13, 2004, pp. 1200-1212.

[22]A. Efors and T. Leung, "Texture synthesis by nonparametric sampling," in Proceedings of the 17th IEEE International Conference on Computer Vision, 1999, pp. 1033-1038.

[23] Y. Chen, Q. Luan, H. Li, O. Au, "Sketch-guided texture-based image inpainting", IEEE trans, pp.4244048,2006.

[24] A. Rares , M. J. T. Reinders, and J. Beimond, "Edgebased image restoration", IEEE trans. On image processing,vol.14, no. 10, pp 1454-1468,oct.2005.

\section{Author Profile}

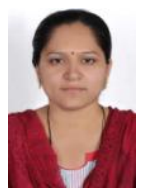

Rohini .B. Late received the B.E. degree in Computer Science and Engineering from M.S. Bidve Engineering College in 2010. Now, she is pursuing Master's in Engineering (Computer Science and Engineering) from M.S. Bidve Engineering college, Latur, SRTM University Nanded, Maharashtra.

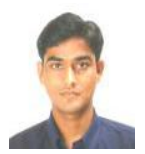

N. G. Dharashive received the B.E. and M.E. degrees in Computer Science \& Engineering from M.B.E.Society's College of Engineering, Ambajogai in 2001 and from Government College of Engineering, Aurangabad in 2011, respectively. He is pursuing Ph.D in Image Processing from S.R.T.M.University, Nanded (M.S.). He is now with M.S.Bidve Engineering College, Latur (M.S.) as Assistant Professor since 2002. 